\title{
Welding fume exposure is associated with inflammation: a global metabolomics profiling study
}

Sipeng Shen ${ }^{1,2,3 \dagger}$, Ruyang Zhang ${ }^{1,2,3 \dagger}$, Jinming Zhang ${ }^{2}$, Yongyue Wei ${ }^{1,3}$, Yichen Guo ${ }^{2}$, Li Su ${ }^{2,3}$, Feng Chen ${ }^{1,3}$ and David C. Christiani ${ }^{2,3,4^{*}}$

\begin{abstract}
Background: Increasing evidence suggests that welding fume exposure is associated with systemic inflammation. Although celluar metabolites may be associated with inflammation, there is limited information on metabolomic changes during welding fume exposure. Such changes may play an important role in the occurrence, development, and prevention of metal-associated diseases. We aim to investigate human metabolomics changes pre- and postwelding fume exposure.
\end{abstract}

Methods: This study included 52 boilermakers totally. We collected plasma samples pre- and post-shift welding fume exposure and prepared samples using the automated MicroLab STAR ${ }^{\circledast}$ system. Metabolite concentrations were measured using ultra performance liquid chromatography - tandem mass spectrometer (UPLC-MS/MS) methods. Two-way analysis of variance was used to test the significance of metabolite changes with false discovery rate correction.

Results: Analysis detected several metabolic changes after welding fume exposure, mainly involved in the lipid pathway [glucocorticoid class (cortisol, corticosterone, and cortisone), acylcarnitine class, and DiHOME species (9,10DiHOME and 12,13-DiHOME)], amino acid utilization (isoleucine, proline and phenylalanine), and S-(3-hydroxypropyl) mercapturic acid (3-HPMA). These compounds are all associated with inflammation according to previous studies. Further, additive interaction effects linked smoking and 3-HPMA levels. In the metabolite set enrichment analysis for diseases, the top two disease-associated metabolite pathways were systemic inflammation-related diseases including rheumatoid arthritis and systemic lupus erythematosus.

Conclusions: This global metabolomics study shows evidence that metabolite changes during welding fume exposure are closely associated with systemic inflammation. The altered metabolites detected may be potential health monitoring biomarkers for boilermakers, especially for inflammation-related disease prevention.

Keywords: Boilermaker, Welding fume exposure, Inflammation, Metabolomics, Occupational health, Environmental exposures

\footnotetext{
*Correspondence: dchris@hsph.harvard.edu

${ }^{\dagger}$ Sipeng Shen and Ruyang Zhang contributed equally to this work.

${ }^{2}$ Department of Environmental Health, Harvard T.H. Chan School of Public Health, Boston, MA 02115, USA

${ }^{3}$ China International Cooperation Center for Environment and Human Health, School of Public Health, Nanjing Medical University, Nanjing 211166, Jiangsu, China

Full list of author information is available at the end of the article
}

(c) The Author(s). 2018 Open Access This article is distributed under the terms of the Creative Commons Attribution 4.0 International License (http://creativecommons.org/licenses/by/4.0/), which permits unrestricted use, distribution, and reproduction in any medium, provided you give appropriate credit to the original author(s) and the source, provide a link to the Creative Commons license, and indicate if changes were made. The Creative Commons Public Domain Dedication waiver (http://creativecommons.org/publicdomain/zero/1.0/) applies to the data made available in this article, unless otherwise stated. 


\section{Background}

Welding fumes comprise a wide range of non-metals and metals with varying toxic effects [1-3]. Epidemiological studies have demonstrated welding fume exposure is associated with various disease, including pulmonary disease, lung inflammation, cardiovascular disease, and cancer $[4,5]$. An improved understanding of possible adverse health effects of exposure to welding fumes, as well as their underlying mechanisms, is important for risk assessment and the development of prevention strategies that will impact a large population of workers [6].

Metabolomics has been increasingly recognized as a powerful functional tool to understand complex biological machinery and to develop new biomarkers for environmental biomonitoring that can help prevent and treat environmental-associated diseases [7]. Metabolomics is based on comprehensive analysis of the endogenous low-molecular-weight biomolecules (typically < $1000 \mathrm{Da}$ ) [8] within a cell, tissue, or biofluid (e.g., plasma or urine) that are associated with different human metabolic processes [7]. Metabolomics applications are expanding in the field of occupational health as a fast and reproducible approach that directly reflects biological events related to exposure [9-12]. Therefore, monitoring disturbances of the metabolome is now more sensitive, easily accessible, less expensive, and more accurate [13].

Despite the availability of such applications, limited research has focused on systemic metabolomics alterations of welding fume exposure. Using the well-established occupational cohort of boilermaker construction workers, we interrogated biochemical profiles manifested in human plasma samples originating from boilermakers with occupational exposure to metal fumes, with the aim of characterizing metabolic migration from pre-exposure to post-exposure. We identified metabolite changes during welding fume exposure and further explored their potential biological functions in boilermakers.

\section{Methods}

\section{Study design and data collection}

We recruited 52 boilermakers at an apprentice welding school (Union Local 29, Quincy, MA) totally. All participants were selected from the well-characterized occupational cohort of boilermaker construction workers in eastern Massachusetts during 2010-2011, as previously described [12, 14]. Peripheral blood samples were collected from all subjects before (pre-) and immediately after (post-) a $\sim 5$-h welding workshop.

According to the welding time, blood sample were drawn in two batches to control the circadian variation [15]. Twenty-nine samples (batch 1) were collected in the morning (pre-) and afternoon (post-) while 23 samples (batch 2) were collected in the afternoon (pre-) and evening (post-) (Table 1). We provided the boilermakers with breakfast, lunch and dinner to control for nutrient intake as a confounder. Samples within each batch were collected in the same time over the same day. Same blood drawers and handling method were used for all samples.

\section{Sample accessioning}

Following receipt, samples were inventoried and maintained at $-80{ }^{\circ} \mathrm{C}$. Each sample was accessioned into the Metabolon Laboratory Information Management System (LIMS) and was assigned by LIMS a unique identifier that was associated with the original source identifier only. This identifier was used to track all sample handling, tasks, results, etc. LIMS tracked all samples and derived aliquots. All portions of any sample were automatically assigned their own unique identifiers by LIMS when a new task was created, and the relationship of these samples was also tracked.

Table 1 Demographic characteristics of the study population

\begin{tabular}{|c|c|}
\hline Characteristic & Mean \pm SD or $N$ \\
\hline Sample size & $52^{a}$ \\
\hline \multicolumn{2}{|l|}{ Sample collecting time } \\
\hline Morning and afternoon & 29 \\
\hline Afternoon and evening & 23 \\
\hline Age (years) & $40.91 \pm 12.22$ \\
\hline Welding time (hours/month) & $33.06 \pm 25.51$ \\
\hline BMI $\left(\mathrm{kg} / \mathrm{m}^{2}\right)$ & $28.43 \pm 5.41$ \\
\hline Weight (kg) & $89.53 \pm 17.89$ \\
\hline Height (m) & $1.77 \pm 0.08$ \\
\hline \multicolumn{2}{|l|}{ Gender } \\
\hline Male & 50 \\
\hline Female & 1 \\
\hline \multicolumn{2}{|l|}{ Race } \\
\hline Caucasian & 43 \\
\hline African American & 4 \\
\hline Asian & 2 \\
\hline Hispanic & 2 \\
\hline \multicolumn{2}{|l|}{ Current smoker } \\
\hline Yes & 21 \\
\hline No & 30 \\
\hline \multicolumn{2}{|l|}{ Medical history } \\
\hline Diabetes & 4 \\
\hline High blood pressure & 5 \\
\hline Irregular heart arrhythmia & 2 \\
\hline High cholesterol hyperlipidemia & 7 \\
\hline
\end{tabular}

Baseline information of one sample was missing 
The purpose of Metabolon LIMS was to enable fully auditable laboratory automation through a secure, easy to use, and highly specialized system. The scope of Metabolon LIMS encompasses sample accessioning, sample preparation, instrumental analysis, reporting, and advanced data analysis. All subsequent software systems were grounded in LIMS data structures. It has been modified to leverage and interface with in-house information extraction and data visualization systems, as well as third-party instrumentation and data analysis software.

\section{Sample preparation}

Samples were prepared using the automated MicroLab STAR system from Hamilton Company. Several recovery standards were added before the first step of the extraction process for $\mathrm{QC}$ purposes. To remove protein, dissociate small molecules bound to protein or trapped in the precipitated protein matrix, and to recover chemically diverse metabolites, proteins were precipitated with methanol under vigorous shaking for $2 \mathrm{~min}$ (Glen Mills GenoGrinder 2000) and then centrifuged. The resulting extract was divided into five fractions: two for analysis by two separate reverse-phase (RP)/ultra-performance liquid chromatography (UPLC)-MS/MS methods with positive ion-mode electrospray ionization (ESI); one for analysis by RP/UPLC-MS/MS with negative ion-mode ESI; one for analysis by HILIC/UPLC-MS/MS with negative ion-mode ESI; and one reserved for backup. Samples were placed briefly on a TurboVap (Zymark) to remove organic solvent. Sample extracts were stored overnight under nitrogen before preparation for analysis.

\section{Instrument variability control}

Instrument variability was determined by calculating the median relative standard deviation (RSD) of internal standards that were added to each sample prior to injection into mass spectrometers ( $\mathrm{RSD}=4 \%$ ). Overall process variability was determined by calculating median RSD for all endogenous metabolites (i.e., non-instrument standards) present in $100 \%$ of pooled matrix samples $(\mathrm{RSD}=8 \%)$. RSD values met Metabolon's acceptance criteria. More details are provided in the Additional file 1.

\section{Pathway enrichment analysis}

To test the statistical significance of pathways, we performed a pathway enrichment analysis based on the hypergeometric test: $P(x=k)=\frac{\left(\frac{K}{k}\right)\left(\frac{N-K}{n-k}\right)}{\frac{N}{n}}$, where $\mathrm{N}$ is the total number of metabolites, $\mathrm{n}$ is the total number of significant metabolites, $\mathrm{K}$ is the number of metabolites in this pathway and $\mathrm{k}$ is number of significant metabolites in this pathway.

\section{Metabolite set enrichment analysis for disease}

To identify and interpret patterns of human metabolite concentration changes with potential diseases, metabolite set enrichment analysis (MSEA) was used based on MetaboAnalyst [16]. We used the library of disease-associated metabolite sets in blood, which contained 416 metabolite sets reported in human blood.

$P$-values for pathway enrichment analysis and MSEA were adjusted by false discovery rate (FDR $q$-value) correction.

\section{Statistical analysis}

In demographic descriptions, mean \pm standard deviation (SD) or frequencies ( $\mathrm{n}$ ) were used to describe continuous variables or categorical variables. We followed these data preprocessing steps: normalization to volume extracted, imputation of missing values with the minimum observed value for each compound [17], and logarithmic transformation. Change of each metabolite during welding day was calculated as fold change value (FC): $\quad F C=\frac{1}{n} \sum_{i=1}^{n}$ $\frac{\text { post-exposure }_{i}}{\text { pre-exposure }_{i}}$, where $i$ represented different subjects. To test the difference of metabolites during exposure and take circadian variation into account, we used two-way analysis of variance (ANOVA) with interaction which included the welding exposure factor and circadian factor. In the results, we reported only the ANOVA results for the exposure effects.

Statistical analyses were performed using $\mathrm{R}$ version 3.3.0 (The $\mathrm{R}$ Foundation). $P$ values were two-sided and FDR correction was calculated to consider multiple comparisons. $F D R-q<0.05$ was considered statistically significant.

\section{Results}

Demographic descriptions of the study population

This study included paired samples (pre-exposure vs. post-exposure) of 52 boilermakers totally. In summary, they had an average age of $40.9 \pm 12.2$ years, ranging from 21 to 63 years; and an average BMI of $28.43 \pm$ $5.41 \mathrm{~kg} / \mathrm{m}^{2}$, ranging from 18 to $39 \mathrm{~kg} / \mathrm{m}^{2}$. Only one individual was female, $42.0 \%$ were current smokers, and $83 \%$ were Caucasians (Table 1). No participants reported metal fever during the welding workshop.

\section{Metabolite alteration summary}

We detected 693 known compounds in 8 metabolite superpathways and 77 subpathways (Additional file 2: Table S1). After quality control, imputation, normalization, and log transformation, we used two-way ANOVA to identify differences between pre-exposure and post-exposure groups. In summary, we identified 113 metabolites were significantly altered-78 were significantly up-regulated after exposure, while 35 were significantly down-regulated. 
Hierarchical clustering could distinguish between pre- and post-exposure workers (Fig. 1). For the circadian variation, 65 metabolites were significantly altered, including 41 up-regulated and 24 down-regulated compounds. However, no metabolites passed FDR correction for the interaction of exposure and circadian effects.

\section{Pathway enrichment analysis results}

We identified 7 of 8 superpathways significantly enriched during the welding exposure, including lipid, amino acid, xenobiotics, carbohydrate, cofactors and vitamins, nucleotide, and energy pathways. For the subpathways, 14 of 41 pathways were significant with $q<0.05$, such as lysolipid, phospholipid metabolism, fatty acid metabolism (acyl carnitine) and steroid pathways (Table 2).

\section{Several classes of significant metabolite alterations}

In the lipid pathway, post-exposure welders exhibited significant decreases in steroid hormones that were mainly involved in the glucocorticoid class [cortisol ( $\mathrm{FC}=0.58 ; q=$

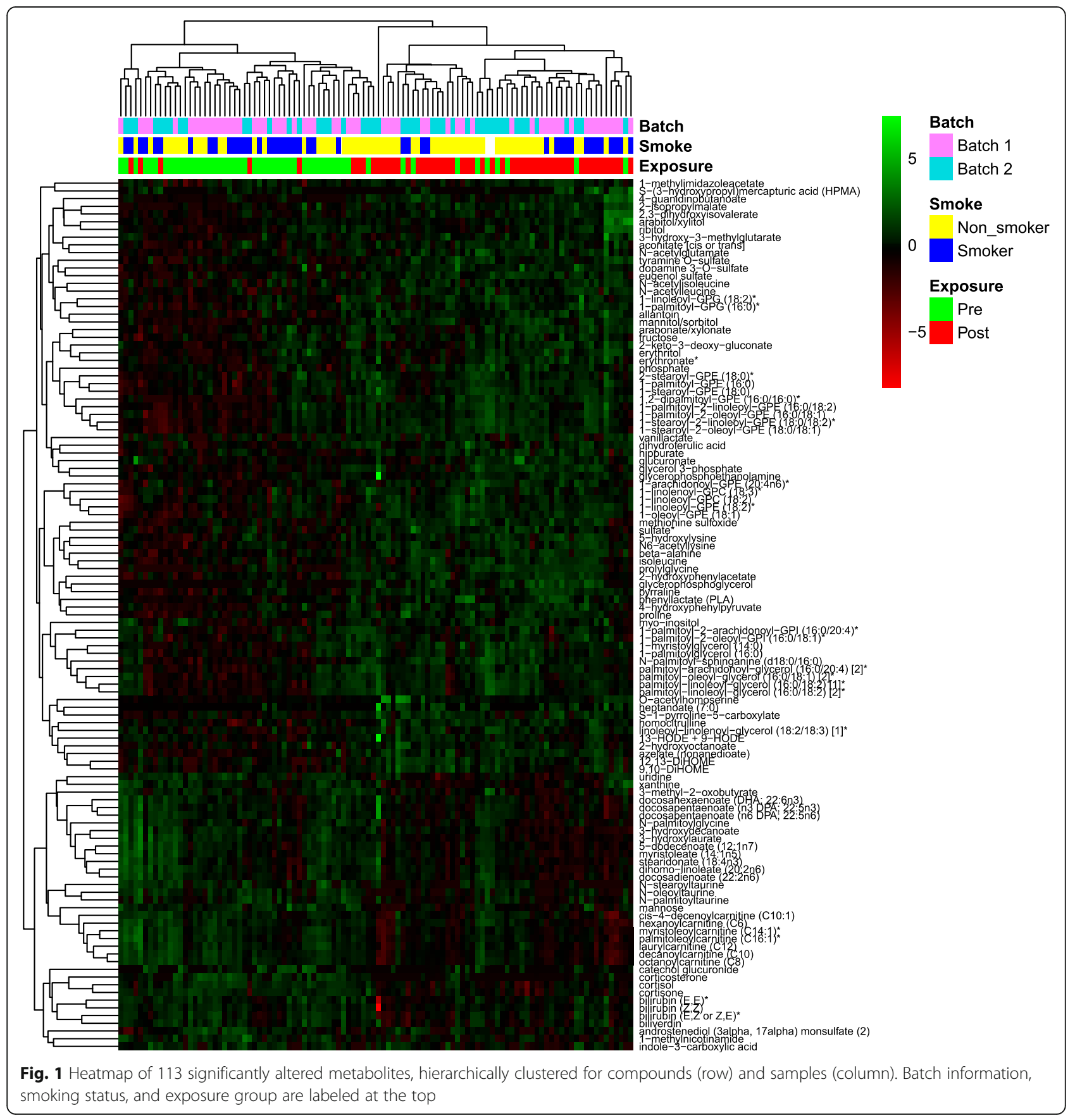


Table 2 Significant superpathways and subpathways in pathway enrichment analysis

\begin{tabular}{|c|c|c|c|c|c|c|c|}
\hline Pathway & Number & Different & Percentage (\%) & $U p^{a}$ & Down $^{a}$ & $P$ & FDR-q \\
\hline \multicolumn{8}{|l|}{ Superpathway } \\
\hline Lipid & 321 & 61 & 19 & 37 & 24 & $2.18 \mathrm{E}-56$ & $1.75 \mathrm{E}-55$ \\
\hline Amino acid & 158 & 22 & 13.92 & 19 & 3 & 7.37E-19 & $2.95 \mathrm{E}-18$ \\
\hline Xenobiotics & 97 & 10 & 8.77 & 10 & 0 & 9.41E-09 & 2.51E-08 \\
\hline Carbohydrate & 21 & 8 & 38.09 & 7 & 1 & 4.04E-07 & $8.08 \mathrm{E}-07$ \\
\hline Cofactors and vitamins & 20 & 5 & 25 & 0 & 5 & $1.07 \mathrm{E}-04$ & $1.71 \mathrm{E}-04$ \\
\hline Nucleotide & 30 & 4 & 13.33 & 2 & 2 & $6.76 \mathrm{E}-04$ & $9.01 \mathrm{E}-04$ \\
\hline Energy & 9 & 2 & 22.22 & 2 & 0 & 2.64E-02 & $3.02 \mathrm{E}-02$ \\
\hline \multicolumn{8}{|l|}{ Subpathway } \\
\hline Lysolipid & 24 & 10 & 41.67 & 10 & 0 & $1.91 \mathrm{E}-07$ & 7.84E-06 \\
\hline Phospholipid Metabolism & 33 & 8 & 24.24 & 8 & 0 & 4.47E-06 & 9.17E-05 \\
\hline Fatty Acid Metabolism (Acyl Carnitine) & 23 & 7 & 30.43 & 0 & 7 & $2.14 \mathrm{E}-05$ & $1.75 \mathrm{E}-04$ \\
\hline Food Component/Plant & 31 & 7 & 22.58 & 7 & 0 & 2.14E-05 & 1.75E-04 \\
\hline Phenylalanine and Tyrosine Metabolism & 29 & 7 & 24.14 & 6 & 1 & $2.14 \mathrm{E}-05$ & $1.75 \mathrm{E}-04$ \\
\hline Polyunsaturated Fatty Acid (n3 and n6) & 13 & 6 & 46.15 & 1 & 5 & $1.02 \mathrm{E}-04$ & $6.94 \mathrm{E}-04$ \\
\hline Diacylglycerol & 19 & 5 & 26.32 & 5 & 0 & 4.79E-04 & 2.80E-03 \\
\hline Fatty Acid, Monohydroxy & 14 & 4 & 28.57 & 2 & 2 & 2.24E-03 & $8.35 \mathrm{E}-03$ \\
\hline Hemoglobin and Porphyrin Metabolism & 5 & 4 & 80.00 & 0 & 4 & 2.24E-03 & $8.35 \mathrm{E}-03$ \\
\hline Leucine, Isoleucine and Valine Metabolism & 24 & 4 & 16.67 & 3 & 1 & 2.24E-03 & $8.35 \mathrm{E}-03$ \\
\hline Steroid & 36 & 4 & 11.11 & 0 & 4 & 2.24E-03 & $8.35 \mathrm{E}-03$ \\
\hline Endocannabinoid & 5 & 3 & 60.00 & 0 & 3 & 1.04E-02 & 3.05E-02 \\
\hline Fructose, Mannose and Galactose Metabolism & 4 & 3 & 75.00 & 2 & 1 & 1.04E-02 & 3.05E-02 \\
\hline Pentose Metabolism & 6 & 3 & 50.00 & 3 & 0 & 1.04E-02 & 3.05E-02 \\
\hline
\end{tabular}

${ }^{a}$ Up indicates higher level in the post-exposure group; down indicates lower level in the post-exposure group

$\left.5.50 \times 10^{-10}\right)$, cortisone $\left(\mathrm{FC}=0.67 ; q=2.43 \times 10^{-8}\right)$, and corticosterone $\left(\mathrm{FC}=0.35 ; q=1.53 \times 10^{-6}\right)$ ] (Fig. 2a). In addition, the post-exposure group showed significant decreases in the acylcarnitine class [hexanoylcarnitine $(\mathrm{FC}=$ $0.81 ; q=0.020)$, decanoylcarnitine $(\mathrm{FC}=0.61 ; q=8.75 \times$ $\left.10^{-5}\right)$, octanoylcarnitine $\left(\mathrm{FC}=0.62 ; q=3.49 \times 10^{-4}\right)$, and laurylcarnitine ( $\mathrm{FC}=0.68 ; q=0.001)$ ] (Fig. 2b). Conversely, the post-exposure group had significantly higher levels of 9,10-DiHOME $\quad\left(\mathrm{FC}=1.87 ; \quad q=4.93 \times 10^{-5}\right) \quad$ and 12,13-DiHOME (FC $=1.91 ; q=1.50 \times 10^{-4}$ ) (Fig. 2c).

In addition to the changes outlined above in the lipid pathway, the post-exposure group also exhibited a number of changes relating to complex lipid homeostasis. There were significant increases in lysolipids (10 of 24), phospholipids (8 of 33) and diacylglycerol (5 of 19) classes which may be related to increased utilization of the fatty acid pool.

Significant increases were observed in amino acid pathway profiles in the post-exposure group, including isoleucine $(\mathrm{FC}=1.19, q=0.008)$ and proline $(\mathrm{FC}=1.18$, $q=0.005)$. Phenylalanine also showed marginally significance $(\mathrm{FC}=1.10, q=0.079)$. Derivatives from these amino acids also significantly increased in the $\mathrm{N}$-acetyl group, such as $\mathrm{N}$-acetylglutamate, $\mathrm{N}$-acetylleucine and $\mathrm{N}$-acetylisoleucine, revealing high amino acid utilization.

Further, post-exposure samples had 2.16-fold increased levels of S-(3-hydroxypropyl) mercapturic acid (3-HPMA) $(q=0.032)$ (Fig. 3a). Considering that 3-HPMA could also be affected through tobacco smoke [18-20], we performed a stratification analysis by smoking status and found 3-HPMA was significantly increased in the smoking group ( $\left.\mathrm{FC}=15.04 ; P=5.74 \times 10^{-7}\right)$ compared to the non-smoking group ( $\mathrm{FC}=1.09 ; P=0.325$ ) (Fig. $3 \mathrm{~b})$. Using two-way ANOVA for welding exposure and smoking status, additive interaction effects were also found between smoking and welding fume exposure $\left(P_{\text {interaction }}=7.56 \times 10^{-10}\right)$.

To exclude the potential physiological confounding caused by sex differences, we performed a sensitivity analysis using only the male workers $(n=50)$. The significance of the altered metabolites remained (Additional file 2: Table S2).

\section{Metabolite set enrichment analysis (MSEA) for disease}

Upon analysis of pre- and post-exposure compounds, MSEA for disease-associated pathways in blood showed 39 significant pathways, including rheumatoid arthritis 


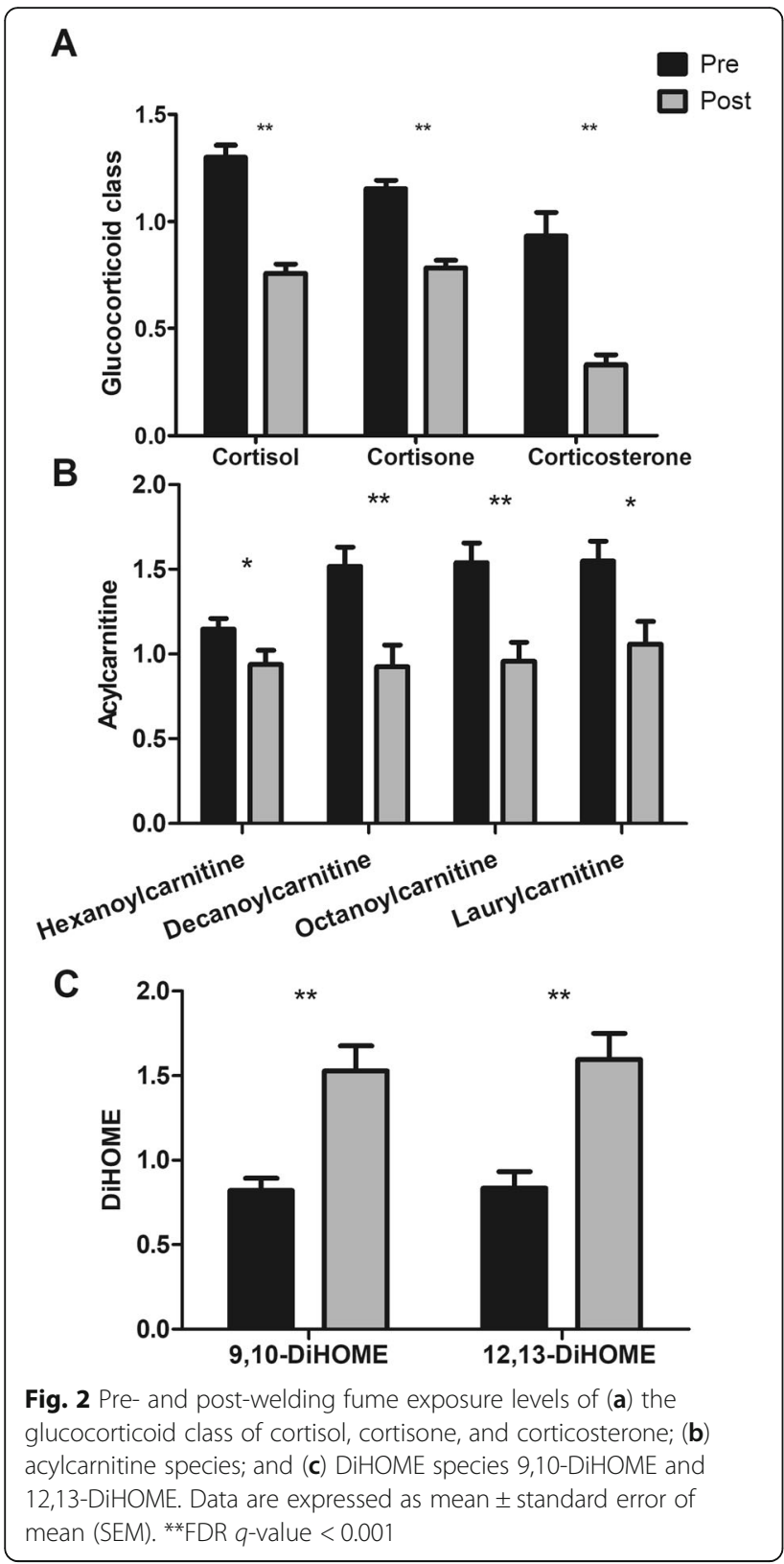

(RA), systemic lupus erythematosus (SLE), and glucocorticoid resistance (Fig. 4, Additional file 2: Table S3).

\section{Discussion}

Recently, studies have reported the relationship between welding fume exposure and inflammation. In this study, we investigated the global metabolomics profiling in human plasma for the first time. Our results revealed some different metabolite classes between exposure groups that were mainly in the lipid pathway (steroid hormones, acylcarnitine class, DiHOME species), amino acid utilization, and 3-HPMA. Interestingly, according to the previous studies [21-26]. These altered compounds showed a close relationship with inflammation, which may indicate an inflammatory mechanism of welding fume exposed boilermakers at the metabolomics level.

We observed significant decreases of glucocorticoid class including cortisol, cortisone and corticosterone as well as their hormone derivatives during welding fume exposure. Glucocorticoids, which belong to the steroid hormones family, are a class involved in regulating glucose metabolism, immunosuppressive, and anti-inflammatory responses in the body [26, 27]. Glucocorticoids are the most effective anti-inflammatory therapy for many chronic inflammatory and immune diseases [25]. Various evidence showed cortisol, cortisone and corticosterone played important roles in the inflammation [28-31]. Thus, monitoring the reductions of the glucocorticoid levels could provide evidence for relevant pulmonary and cardiovascular diseases.

We found significant decreases in the acylcarnitine class after exposure to welding fumes. The acylcarnitine class of metabolites often tracks with free fatty acids, as they are formed when fatty acids conjugate with carnitine. As important lipid biomarkers reflective of acyl-CoA status, acylcarnitines possess bioactive and inflammatory properties. Acylcarnitines have been reported as a marker of incomplete fatty acid $\beta$-oxidation and mitochondrial dysfunction [32]. In addition, alteration of acylcarnitines has an activating effect in many proinflammatory signaling pathways, and thus the compounds might have the potential to activate inflammation [24]. Acylcarnitines are also in insulin-resistance development, linking to muscle oxidative stress and inflammation [33]. Additionally, inflammatory bowel disease patients also have significantly lower acylcarnitine levels [34]. These results provide new evidence for an association with inflammatory diseases.

9,10-DiHOME and 12,13-DiHOME are derived from linoleate through epoxidation and hydration reactions and are thought to participate in mediating inflammatory responses [23]. They have been implicated in inflammatory disorders, such as adult respiratory distress syndrome [35], asthma [36], and may be endogenous regulators of vascular permeability and inflammation [37]. Their increased levels following welding fume exposure may provide new evidence for the association between DiHOME levels and systemic inflammatory responses [38].

Metabolic changes associated with inflammatory processes and immune responses can modify protein and amino acid requirements [39]. Such changes are usually considered the consequence of an increase in the production of cytokines that change protein metabolism. During the immunological stress process, amino acids are redistributed away from protein production towards tissues involved in inflammation and immune response [39]. We found that some groups of amino acids increased after exposure to welding fumes, which was consistent with a 

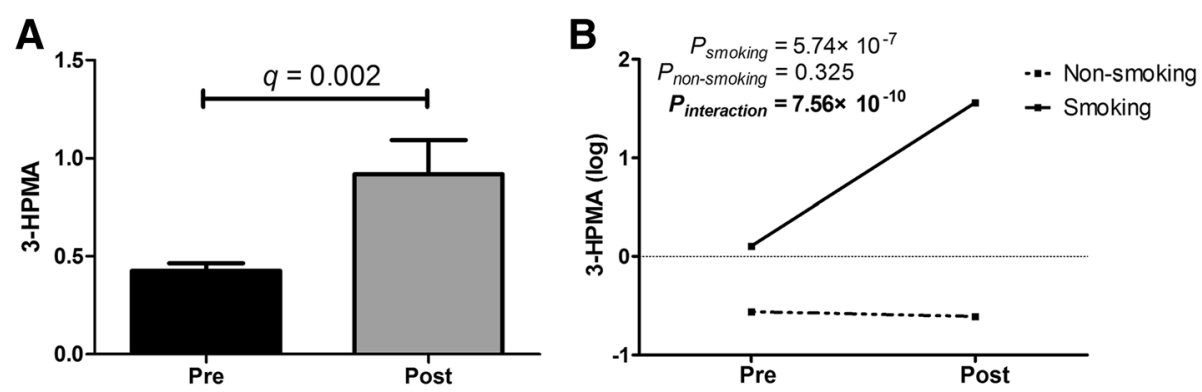

Fig. 3 a Pre- and post-welding fume exposure levels of 3-HPMA. Data are expressed as mean \pm SEM. $\mathbf{b}$ Interaction plot of 3-HPMA levels and smoking status for pre- and post-welding fume exposure groups. $P_{\text {smoking }}$ indicates $t$-test $P$-value of 3-HPMA within the smoking subgroup, while $P_{\text {non-smoking }}$ indicates $P$-value within the non-smoking subgroup. Two-way analysis of variance was used based on log-transformed data. $P_{\text {interaction }}$ indicates $P$-value of interaction effects

report that utilization of some amino acids increases during chronic inflammation [21].

3-HPMA is regarded as one of the main metabolites of acrolein, which is a toxic by-product of unsaturated aldehyde [40]. It is also a reliable biomarker to estimate acrolein concentration [41]. Acrolein exposure can occur through several mechanisms, including welding, particularly when the work involves painted materials and/or anticorrosive primers [42]. Main endogenous sources of acrolein are degradation of amino acids and polyamines, which may constitute a significant source of acrolein in situations of oxidative stress and inflammation [40]. Nevertheless, 3-HPMA levels are positively associated with cigarette smoking $[19,43]$. We also found additive interactions between 3-HPMA and smoking that provide new evidence for further investigation of welding fume exposure and smoking-induced disease.
MSEA for disease linked metabolites with altered expression in the post exposure samples to different sets showed associations with various diseases. Of them, the top two disease-associated metabolite pathways were systemic inflammation-related diseases RA and SLE; the interaction was related to decreased cortisol and cortisone from steroid hormones. Several studies have reported that exposure to welding fumes containing metal is associated with systemic inflammation [44-46]. Cortisol and cortisone production are related to chronic inflammatory diseases, including RA and SLE [47], while welding fumes have also been reported as risk factors for RA [48, 49] and SLE [50]. Further, $\mathrm{C}$-reactive protein (CRP) is considered a strong marker for systemic inflammation [51], and positive correlations are found within CRP and cortisol [52]. These results provide new evidence for an association between welding fumes and systemic inflammation at the metabolomics level.

Rheumatoid Arthritis
Systemic Lupus Erythematosus
Lipoid Adrenal Hyperplasia (Star Deficiency)
17-Alpha-Hydroxylase Deficiency (Cyp17)
Metabolites Affected By Diurnal Variation
Female Aging After Menopause
Glucocorticoid Resistance
Congenital Adrenal Hyperplasia
Anorexia Nervosa


We found several specific metabolites associated with inflammation, which may be potential health monitoring biomarkers for boilermakers, especially for inflammation-related diseases. Thus, health care products to boost immunity (e.g. vitamins) or anti-inflammatory could be useful in the boilermakers at risk to prevent the systemic inflammation and its consequences diseases.

However, we also recognize some limitations in the study. Firstly, though we considered circadian variation, this only reflected the differences between individuals at different collecting times, but not within individual variation. Secondly, we lacked important inflammation associated markers (e.g. CRP, white blood cell count, interleukin 6) to verify the relationship between metabolites and inflammation. Further studies are needed to validate the associations.

\section{Conclusions}

In summary, this global metabolomics study shows the altered compounds including steroid hormones, acylcarnitine and DiHOME levels during welding fume exposure are associated with systemic inflammatory processes. The identified significant altered metabolites may be potential biomarkers for exposure-related inflammatory diseases among the boilermakers.

\section{Additional files}

Additional file 1: Supplementary materials and methods. Table S4. Description of Metabolon QC samples. Table S5. Metabolon QC standards. Table S6. Data quality: Instrument and process variability. Figure S1. Preparation of technical replicates. (DOCX $120 \mathrm{~kb}$ )

Additional file 2: Table S1. Statistical summary of the 693 metabolites. Table S2.Sensitivity analysis that only keep the male workers. Table S3. MSEA analysis for disease-associated metabolite sets (blood). (XLSX 318 kb)

\section{Abbreviations}

3-HPMA: S-(3-hydroxypropyl) mercapturic acid; ANOVA: Analysis of variance; CRP: C-reactive protein; FC: Fold change; FDR: False discovery rate; LIMS: Laboratory information management system; MSEA: Metabolite set enrichment analysis; RA: Rheumatoid arthritis; RSD: Relative standard deviation; SD: Standard deviation; SLE: Systemic lupus erythematosus

\section{Acknowledgements}

We thank all the participants in our study. The authors would like to thank Angela Fan, Cali Chang, Mike Wang, Rachel Grashow and Rihong Zhai and the International Brotherhood of Boilermakers, Local 29, Quincy MA, USA.

\section{Funding}

The authors thank all participants enrolled in this study. This work was supported by the National Institutes of Health and the National Institute for Environmental Health Sciences (R01ES009860 and P30ES000002 to DCC) and partly supported by the National Institute for Occupational Safety and Health (T42 OH008416 to DCC) and the National Natural Scientific Funding of China (81473070 and 81530088 to FC), China Postdoctoral Science Foundation (2018M633767 to RZ), Natural Science Foundation of the Jiangsu Higher Education Institutions of China (18KJB310011 to RZ and 14KJA310002 to FC). The work was also supported by Nanjing Medical University international exchange and cooperation project (C018 to SS). RZ and YW were partially supported by the Outstanding Young Teachers Training Program of Nanjing
Medical University. Sponsors had no role in study design, data collection and analysis, decision to publish, or preparation of the manuscript.

\section{Availability of data and materials}

Please contact author for data requests.

\section{Authors' contributions}

Conceived and designed experiments: SS, RZ, and DCC. Performed experiments: SS, JZ, LS, and DCC. Analyzed data: SS. Contributed reagents/ materials/analysis tools: SS and FC. Wrote the paper: SS. Acquired data: LS. Supervised the study: DCC. Collected data: JZ. Obtained funding for study: DCC. All authors read and approved the final manuscript.

\section{Ethics approval and consent to participate}

The Institutional Review Board at the Harvard School of Public Health approved the study protocol, and informed written consent was obtained from each participant.

Consent for publication

Not applicable.

\section{Competing interests}

The authors declare that they have no competing interests.

\section{Publisher's Note}

Springer Nature remains neutral with regard to jurisdictional claims in published maps and institutional affiliations.

\section{Author details}

'Department of Biostatistics, School of Public Health, Nanjing Medical University, Nanjing 211166, Jiangsu, China. ${ }^{2}$ Department of Environmental Health, Harvard T.H. Chan School of Public Health, Boston, MA 02115, USA.

${ }^{3}$ China International Cooperation Center for Environment and Human Health School of Public Health, Nanjing Medical University, Nanjing 211166, Jiangsu, China. ${ }^{4}$ Department of Medicine, Pulmonary and Critical Care Division, Massachusetts General Hospital and Harvard Medical School, 665 Huntington Avenue, Building I Room 1401, Boston, MA 02115, USA.

Received: 28 December 2017 Accepted: 9 August 2018

Published online: 22 August 2018

References

1. Antonini JM. Health effects of welding. Crit Rev Toxicol. 2003;33(1):61.

2. Antonini JM, Lewis $A B$, Roberts JR, Whaley DA. Pulmonary effects of welding fumes: review of worker and experimental animal studies. Am Ind Med. 2003:43(4):350-60

3. Erdely A, Salmen-Muniz R, Liston A, Hulderman T, Zeidler-Erdely PC, Antonini JM, et al. Relationship between pulmonary and systemic markers of exposure to multiple types of welding particulate matter Toxicology. 2011;287(1):153-9.

4. Guha N, Loomis D, Guyton KZ, Grosse Y, El Ghissassi F, Bouvard V, Benbrahim-Tallaa L, Vilahur N, Muller K, Straif K. Carcinogenicity of welding, molybdenum trioxide, and indium tin oxide. The Lancet Oncology. 2017; 18(5):581-2.

5. Ibfelt E, Bonde JP, Hansen J. Exposure to metal welding fume particles and risk for cardiovascular disease in Denmark: a prospective cohort study. Occup Environ Med. 2010;67(11):772.

6. Baumann R, Joraslafsky S, Markert A, Rack I, Davatgarbenam S, Kossack V, et al. IL-6, a central acute-phase mediator, as an early biomarker for exposure to zinc-based metal fumes. Toxicology. 2016;373

7. García-Sevillano MÁ, García-Barrera T, Gómez-Ariza JL. Environmental metabolomics: biological markers for metal toxicity. Electrophoresis. 2015:36(18):2348-65.

8. Bowen BP, Northen TR. Dealing with the unknown: metabolomics and metabolite atlases. J Am Soc Mass Spectrom. 2010;21(9):1471-6.

9. Wang SY, Yu CP, Pan YL, Zhou XR, Rui X, Yue W, et al. Metabolomics analysis of serum from subjects after occupational exposure to acrylamide using UPLC-MS. Mol. Cell. Endocrinol. 2017:444:67-75.

10. Walker DI, Uppal K, Zhang L, Vermeulen R, Smith M, Hu W, et al. Highresolution metabolomics of occupational exposure to trichloroethylene. Int J Epidemiol. 2016;45(5):1517-27. 
11. Baker M, Simpson C, Lin Y, Seixas N. S15-3 Use of metabolomics in characterising occupational exposures to manganese in metalworkers. Occup Environ Med. 2016;73(Suppl 1):A118.2-A.

12. Wei Y, Wang Z, Chang C, Fan T, Su L, Chen F, et al. Global metabolomic profiling reveals an association of metal fume exposure and plasma unsaturated fatty acids. PLoS One. 2013;8(10):e77413.

13. Bugrim A, Nikolskaya T, Nikolsky Y. Early prediction of drug metabolism and toxicity: systems biology approach and modeling. Drug Discov Today. 2004;9(3):127-35.

14. Chen JC, Stone PH, Christiani DC. Obesity is a modifier of autonomic cardiac responses to fine metal particulates. Environ Health Perspect. 2007;115(7):1002.

15. Zhang J, Fang SC, Mittleman MA, Christiani DC, Cavallari JM. Secondhand tobacco smoke exposure and heart rate variability and inflammation among non-smoking construction workers: a repeated measures study. Environ Health. 2013;12(1):83.

16. Xia J, Wishart DS. Web-based inference of biological patterns, functions and pathways from metabolomic data using MetaboAnalyst. Nat Protoc. 2011;6(6):743.

17. Zimring JC, Slichter S, Odem-Davis K, Felcyn JR, Kapp LM, Bell LN, et al. Metabolites in stored platelets associated with platelet recoveries and survivals. Transfusion. 2016;56(8):1974-83.

18. Hecht SS, Carmella SG, Kotandeniya D, Pillsbury ME, Chen M, Ransom BW, et al. Evaluation of toxicant and carcinogen metabolites in the urine of ecigarette users versus cigarette smokers. Nicotine Tob Res. 2014;17(6):704-9.

19. Carmella SG, Chen M, Zhang Y, Zhang S, Hatsukami DK, Hecht SS. Quantitation of acrolein-derived (3-hydroxypropyl) mercapturic acid in human urine by liquid chromatography- atmospheric pressure chemical ionization tandem mass spectrometry: effects of cigarette smoking. Chem Res Toxicol. 2007;20(7):986-90.

20. McRobbie H, Phillips A, Goniewicz ML, Smith KM, Knight-West O, Przulj D, et al. Effects of switching to electronic cigarettes with and without concurrent smoking on exposure to nicotine, carbon monoxide, and acrolein. Cancer Prev Res. 2015;8(9):873-8.

21. Melchior D, Sève B, Floc'h L. Chronic lung inflammation affects plasma amino acid concentrations in pigs 1. J Anim Sci. 2004;82(4):1091-9.

22. Liu J, Liang Q, Frost-Pineda K, Muhammad-Kah R, Rimmer L, Roethig H, et al. Relationship between biomarkers of cigarette smoke exposure and biomarkers of inflammation, oxidative stress, and platelet activation in adult cigarette smokers. Cancer Epidemiol Prev Biomarkers. 2011;20(8):1760-9.

23. Gouveia-Figueira S, Späth J, Zivkovic AM, Nording ML. Profiling the oxylipin and endocannabinoid metabolome by UPLC-ESI-MS/MS in human plasma to monitor postprandial inflammation. PLoS One. 2015;10(7):e0132042.

24. Rutkowsky JM, Knotts TA, Ono-Moore KD, McCoin CS, Huang S, Schneider D, et al. Acylcarnitines activate proinflammatory signaling pathways. Am J Physiol Endocrinol Metab. 2014;306(12):E1378-E87.

25. Barnes PJ. Mechanisms and resistance in glucocorticoid control of inflammation. J Steroid Biochem Mol Biol. 2010;120(2-3):76-85.

26. Yeager MP, Rassias AJ, Fillinger MP, DiScipio AW, Gloor KE, Gregory JA, et al. Cortisol antiinflammatory effects are maximal at postoperative plasma concentrations. Crit Care Med. 2005;33(7):1507-12.

27. Koper JW, van Rossum EF, El VDA. Glucocorticoid receptor polymorphisms and haplotypes and their expression in health and disease. Steroids. 2014;92(92):62-73.

28. Oaks B, Stewart C, Laugero K, Adu-Afarwuah S, Lartey A, Baldiviez L, et al. Associations of maternal cortisol, inflammation, hemoglobin, iron status, and BMI with birth outcomes in pregnant women in Ghana. FASEB J. 2015;29(1 Supplement):579.1.

29. Dougherty TF, Schneebell GL. Role of cortisone in regulation of inflammation. Proc Soc Exp Biol Med. 1950;75(3):854-9.

30. de Oliveira APL, Oliveira-Filho RM, da Silva ZL, Borelli P, de Lima WT. Regulation of allergic lung inflammation in rats: interaction between estradiol and corticosterone. Neuroimmunomodulation. 2004;11(1):20-7.

31. Sebastiano M, Eens M, Angelier F, Pineau K, Chastel O, Costantini D. Corticosterone, inflammation, immune status and telomere length in frigatebird nestlings facing a severe herpesvirus infection. Conserv Physiol. 2017;5(1).

32. Koves TR, Ussher JR, Noland RC, Slentz D, Mosedale M, Ilkayeva O, et al. Mitochondrial overload and incomplete fatty acid oxidation contribute to skeletal muscle insulin resistance. Cell Metab. 2008;7(1):45-56.

33. Aguer C, McCoin CS, Knotts TA, Thrush AB, Ono-Moore K, McPherson R, et al. Acylcarnitines: potential implications for skeletal muscle insulin resistance. FASEB J. 2015;29(1):336-45.
34. Danese C, Cirene M, Colotto M, Aratari A, Amato S, Di Bona S, et al. Cardiac involvement in inflammatory bowel disease: role of acylcarnitine esters. La Clinica Terapeutica. 2010;162(4):e105-9.

35. Smith KR, Pinkerton KE, Watanabe T, Pedersen TL, Ma SJ, Hammock BD. Attenuation of tobacco smoke-induced lung inflammation by treatment with a soluble epoxide hydrolase inhibitor. Proc Natl Acad Sci U S A. 2005;102(6):2186-91.

36. Levan SR, Fujimura KE, Lin D, Lukacs NW, Ownby DR, Johnson CC, et al. The childhood asthma-associated metabolite 12, 13 DiHOME, suppresses regulatory T cells. J Allergy Clin Immunol. 2017;139(2):AB84.

37. Slim R, Hammock BD, Toborek M, Robertson LW, Newman JW, Morisseau $\mathrm{CH}$, et al. The role of methyl-linoleic acid epoxide and diol metabolites in the amplified toxicity of linoleic acid and polychlorinated biphenyls to vascular endothelial cells. Toxicol Appl Pharmacol. 2001;171(3):184-93.

38. Yu IJ, Song KS, Maeng SH, Kim SJ, Sung JH, Han JH, et al. Inflammatory and genotoxic responses during 30-day welding-fume exposure period. Toxicol Lett. 2004;154(1):105-15.

39. Le Floc'h N, Melchior D, Obled C. Modifications of protein and amino acid metabolism during inflammation and immune system activation. Livest Prod Sci. 2004;87(1):37-45.

40. Yan W, Byrd GD, Brown BG, Borgerding MF. Development and validation of a direct LC-MS-MS method to determine the acrolein metabolite 3-HPMA in urine. J Chromatogr Sci. 2010;48(3):194-9.

41. Zheng L, Park J, Walls M, Tully M, Jannasch A, Cooper B, et al. Determination of urine 3-HPMA, a stable acrolein metabolite in a rat model of spinal cord injury. J Neurotrauma. 2013;30(15):1334

42. Engström K, Engström B, Henrikseckerman ML. Evaluation of exposures during the welding or flame-cutting of painted steel. Scand J Work Environ Health. 1988;14(4):33-4.

43. Mascher DG, Mascher HJ, Scherer G, Schmid E. High-performance liquid chromatographic-tandem mass spectrometric determination of 3hydroxypropylmercapturic acid in human urine. J Chromatogr B Biomed Sci Appl. 2001;750(1):163-9.

44. Brand P, Bauer M, Gube M, Lenz K, Reisgen U, Spiegel-Ciobanu VE, et al. Relationship between welding fume concentration and systemic inflammation after controlled exposure of human subjects with welding fumes from metal inert gas brazing of zinc-coated materials. J Occup Environ Med. 2014;56(1):1-5.

45. Markert A, Baumann R, Gerhards B, Gube M, Kossack V, Kraus T, et al. Single and combined exposure to zinc-and copper-containing welding fumes lead to asymptomatic systemic inflammation. J Occup Environ Med. 2016:58(2):127-32.

46. Kauppi $P$, Järvelä $M$, Tuomi $T$, Luukkonen $R$, Lindholm $T$, Nieminen $R$, et al Short-term respiratory and systemic inflammatory responses to welding exposure. Eur Respiratory Soc. 2015;46(suppl 59), PA1152.

47. Straub R, Weidler C, Demmel B, Herrmann M, Kees F, Schmidt M, et al. Renal clearance and daily excretion of cortisol and adrenal androgens in patients with rheumatoid arthritis and systemic lupus erythematosus. Ann Rheum Dis. 2004;63(8):961-8.

48. Hutchinson D. E32. The Rise and Fall of Seropositive Rheumatoid Arthritis: Exposure to Cadmium is a Plausible Explanation for the Natural History of Seropositive Rheumatoid Arthritis. Rheumatology. 2015;54(suppl 1):i184-i.

49. Roos AJD, Cooper GS, Alavanja MC, Sandler DP. Rheumatoid arthritis among women in the agricultural health study: risk associated with farming activities and exposures. Ann Epidemiol. 2004;15(10):762-70.

50. Parks CG, De Roos AJ. Pesticides, chemical and industrial exposures in relation to systemic lupus erythematosus. Lupus. 2014;23(6):527-36.

51. Koenig $W$, Sund $M$, Fröhlich $M$, Fischer $H-G$, Löwel $H$, Döring $A$, et al. C-reactive protein, a sensitive marker of inflammation, predicts future risk of coronary heart disease in initially healthy middle-aged men. Circulation. 1999:99(2):237-42.

52. Dugue B, Leppänen E, Teppo FA-M, Fyhrquist F, Gräsbeck R. Effects of psychological stress on plasma interleukins-1 beta and 6, C-reactive protein, tumour necrosis factor alpha, anti-diuretic hormone and serum cortisol. Scand J Clin Lab Invest. 1993:53(6):555-61. 